\title{
DLIGAND2: an improved knowledge-based energy function for protein-ligand interactions using the distance-scaled, finite, ideal-gas reference state
}

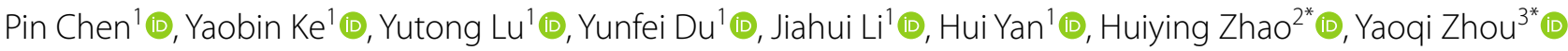 \\ and Yuedong Yang ${ }^{1 *}$ (D)
}

\begin{abstract}
Performance of structure-based molecular docking largely depends on the accuracy of scoring functions. One important type of scoring functions are knowledge-based potentials derived from known three-dimensional structures of proteins and/or protein-ligand complex structures. This study seeks to improve a knowledge-based protein-ligand potential based on a distance-scale finite ideal-gas reference (DFIRE) state (DLIGAND) by expanding the representation of protein atoms from $13 \mathrm{~mol} 2$ atom types to 167 residue-specific atom types, and employing a recently updated dataset containing 12,450 monomer protein chains for training. We found that the updated version DLIGAND2 has a consistent improvement over DLIGAND in predicting binding affinities for either native complex structures or docking-generated poses. More importantly, DLIGAND2 has a 52\% increase over DLIGAND in enrichment factors in top $1 \%$ predictions based on the DUD-E decoy set, and consistently improves over Autodock Vina and other statistical energy functions in all three benchmark tests. We further found that DLIGAND2 outperforms empirical and machinelearning methods compared for virtual screening on new targets that are not homologous to the DUD-E training set. Given the best performance as a parameter-free statistical potential and among the best in all performance measures, DLIGAND2 should be useful for re-assessing the poses generated by docking software, or acting as one term in other scoring functions. The program is available at https://github.com/sysu-yanglab/DLIGAND2.
\end{abstract}

Keywords: Docking, Protein-ligand interaction, Knowledge-based energy function

\section{Introduction}

Structure-based molecular docking is one of the key components in computer-aided drug design [1-3]. Docking is a two-step process: conformational sampling of ligands bound to their receptors, followed by assessment of binding free energy between them. Due to advances in

\footnotetext{
*Correspondence: zhaohy8@mail.sysu.edu.cn; yaoqi.zhou@griffith.edu.au; yangyd25@mail.sysu.edu.cn

${ }^{1}$ National Supercomputer Center in Guangzhou, School of Data and Computer Science, Sun Yat-sen University, 132 East Circle at University City, Guangzhou 510006, China

2 Sun Yat-sen Memorial Hospital, Sun Yat-sen University, Guangzhou 510000, China

${ }^{3}$ Institute for Glycomics and School of Information and Communication Technology, Griffith University, Southport, QLD 4215, Australia
}

computing power and numerical algorithms, the success of docking is no longer restricted by inadequacy of conformational sampling but limited instead by the lack of a precise and reliable scoring function to evaluate the free energy of interactions between proteins and ligands [4]. Developing an accurate scoring function is challenging because molecular interaction is contributed by a delicate balance between several different types of interactions including van der Waals and columbic interactions in between, and interactions with solvent environment in addition to the difficulty in capturing entropic contributions $[5,6]$.

A wide variety of scoring functions has been developed to approximate energy functions. Based on the derivation 
ways, scoring functions are usually classified into physics-based methods, empirical scoring functions, knowledge-based potentials, and descriptor-based scoring functions [7]. Physics-based methods, widely employed in molecular dynamics simulation studies, are obtained by combing quantum mechanical calculations of small molecular fragments and empirical fitting to known experimental data. Some examples are linear interaction energy (LIE) [8, 9], linear response approximation (LRA) [10] and MM-PBSA/GBSA [11-13]. Since this type of methods require intensive computing time to perform kinetic integration for entropic effects, they are limited to assess a small number of compounds. Differently, virtual screening usually docked millions of molecules into a protein receptor to locate active compounds. Thus, the requirement of fast computation leads to the dominance of computationally efficient empirical scoring functions in docking as shown in the score-function assessment $[5,6]$. Empirical scoring functions are based on a linear combination of various energetic terms to approximate binding free energy. Notable examples are ChemScore $[14,15]$, X-Score [16], Glide-Score [17, 18], and etc. Typically, the weight factors for individual energetic terms in an empirical scoring function are obtained by regression to achieve the highest correlation to experimental binding affinities (scoring power). More recently, machine learning methods have been used to combine energetic terms and/or employ protein-ligand distances for training. Examples are RF-Score [19], ID-Score [20], SVM-SP [21], and DrugVQA [22]. However, these scoring functions are often sensitive to docking poses and don't perform well to separate decoys from true binding ligands in actual docking experiments [23]. Knowledge-based potentials (or statistical potentials) are derived from statistical analysis of known protein structures. A typical knowledge-based potential considers only the distances between atom pairs that allow efficient calculations. Different knowledge-based functions differ in how proteinligand atom pair potentials and their reference states are defined. Examples are SmoG [24, 25], DrugScore [26], IT-Score [27, 28], and ASP [29]. Knowledge-based scoring functions are also used in combination with solvation and entropic terms to improve performance. Examples are DSX [30], SmoG2016 [31] and ITScore/SE [32].

Previously, a knowledge-based scoring function called DLIGAND [9] was developed based on the distancescaled finite ideal-gas reference (DFIRE) state [33, 34], which has successfully been used for protein interactions with DNA [35], RNA [36], and carbohydrate [37] molecules. DLIGAND was developed by representing both protein and ligand atoms by a few mol 2 atom types, and trained on a small set of 200 protein complex structures. Here, we developed DLIGAND2 by substituting $13 \mathrm{~mol} 2$ atom types by 167 residue-specific atom types for protein atoms and using a large protein structural dataset for training. We showed that DLIGAND2 not only significantly improves over DLIGAND but also has superior performance in separating true ligands from decoys in Database of Useful Decoys-Enhanced (DUD-E).

\section{Methods \\ Scoring function DLIGAND2 potential}

We have used the same approach as the DLIGAND [38] to derive the distance-dependent interaction energy function between atomic pairs based on the distancescale finite ideal-gas reference (DFIRE) state [33] as

$$
\bar{\mu}(i, j, r)=\left\{\begin{array}{cl}
-\eta R T \ln \frac{N_{o b s}(i, j, r)}{\left(\frac{r}{r_{c u t}}\right)^{\alpha}\left(\frac{\Delta r}{\Delta r_{c u t}}\right) N_{o b s}\left(i, j, r_{c u t}\right)}, & r<r_{\text {cut }} \\
0, & r \geq r_{\text {cut }}
\end{array}\right.
$$

where $\mathrm{R}$ is the gas constant, $\mathrm{T}=300 \mathrm{~K}, \alpha=1.61$, $r_{c u t}=15 \AA, \eta$ is a scaling factor simply set as $0.01 / \mathrm{RT}$. $\mathrm{N}_{\text {obs }}(\mathrm{i}, \mathrm{j}, \mathrm{r})$ is the number of atomic pair $(\mathrm{i}, \mathrm{j})$ within the spherical shell of distance $r$ observed in a given structure database, and $\Delta r\left(\Delta r_{\text {cut }}\right)$ is the bin width at $r\left(r_{\text {cut }}\right)$. A constant value of $0.5 \AA$ was used for $\Delta \mathrm{r}$ at all bins and $\Delta \mathrm{r}_{\text {cut }}=\Delta \mathrm{r}$. Here, we employed residue-specific atomic types for protein atoms that leads to 167 atomic types for protein atoms. This is different from DLIGAND, where both protein and ligand atoms were represented by mol2 atom types, and thus only 13 atom types were utilized for protein atoms.

We derived the protein-ligand interactions from protein structures because there is only a small number of non-redundant protein-ligand complex structures. From protein structures, we obtained the $\mathrm{N}_{\mathrm{obs}}$ for the number of observed pairs between protein atoms, which are converted to protein-ligand interactions by mapping indices for protein atoms to $11 \mathrm{~mol} 2$ atom types (see Additional file 1: Table S1) and summing over all pairs that are mapped to the same mol2 atom type as

$$
N_{o b s}^{\prime}(i, k, r)=\sum_{j} N_{o b s}(i, j, r) \delta(\operatorname{map}(j), k),
$$

where $i$ is protein atom type, $\delta(\operatorname{map}(j), k)$ is 1 only when the protein atom type $j$ is mapped to mol 2 atom type $k$, otherwise 0 . Based on the $N_{o b s}^{\prime}(i, k, r)$, we can derive the potential function in the same manner as DFIRE. This design enables us to obtain the scoring function purely from protein atoms without requiring their binding partners, so we employed our recently collected 12,450 non-redundant protein monomer chains [39] to obtain a sufficient number of observations. This training set represents more than 60 times bigger than the dataset (195 
complex structures) used for deriving DLIGAND. For ligand mol2 atom types not existed in proteins, they were mapped to the closest atom type, as detailed in Additional file 1: Table S1. We also adopted the low-count correction according to Bayesian statistics as the previous study [40].

\section{Benchmark datasets}

Four benchmark datasets were employed to evaluate DLIGAND2. The first dataset is CASF-2013 [5, 6], a widely used benchmark containing 195 representative protein-ligand complexes. This benchmark has been used to test the accuracy of binding affinity prediction by using experimentally determined protein-ligand complex structures. The second dataset is the PDBbind refined set (version 2016) [41] of 4057 protein-ligand interaction pairs with experimentally measured binding affinity data. We generated protein-ligand complex structures by docking ligands onto their corresponding receptors respectively with eight docking packages, including AudoDock (version 4.2.6) [42], AutoDock Vina (version 1.1.2) [43], rDock (version 2013.1) [44], LeDock (version 1.0) [45], UCSF DOCK (version 6.8) [46], iDock (version 2013.1) [47], GalaxyDock (with BP2 Score) [48, 49], and iGEMDOCK (version 2.1) [50]. Docking ligands are confined to a $10 \AA$ box enclosing the centroid of co-crystalized ligand. The maximum number of docking poses for each ligand was set to 10 . After removing complexes failing to yield any complex structures in our selected docking programs, a collection of 4044 complexes remained for evaluation. The full list of $4044 \mathrm{com}-$ plexes can be found in Additional file 2: Table S2. The scoring ability of functions were evaluated by the Pearson correlation coefficient (PCC) between the predicted and experimental values, as well as the root mean squared error (RMSE) after linear regression.

The ability of DLIGAND2 to perform virtual screening was also evalued on the DUD-E dataset [51]. There are 22, 886 active ligands binding with 102 targets, with an average of 224 ligands per target. For each target, the DUD-E database provides an abundant number of decoys (50 decoys for each active) that have similar physicalchemical properties but dissimilar two-dimensional (2D) topology. We employed the 3D structure of a target protein with the highest resolution in the protein data bank for docking. This is different from original DUD-E test where the 3D structure of the best performance was selected for each target [51]. For each pair of protein target and ligand compound, we employed Autodock Vina with default options to generate one pose, which are re-scored by 5 scoring functions $\left(\Delta\right.$ vinaRF $F_{20}$, ID-Score, $\mathrm{X}$-Score, DLIGAND, and DLIGAND2).
The accuracy of each scoring function was evaluated by the LogAUC and enrichment factor (EF).

As described in DUD-E Ref. [51] and our previous studies [52, 53], LogAUC takes the logarithm of $x$-axis in area under curve (AUC) to show more information on enrichment at a low false positive rate. We chose three regions of $\mathrm{EF}$ in top $x \%$ of the DUD-E dataset, where $x$ equals to 1,5 and 10 respectively.

$$
E F^{x \%}=\frac{N_{\text {True }}^{x \%} / N_{\text {Selected }}^{x \%}}{N_{\text {Active }} / N_{\text {Total }}}
$$

where $N_{\text {True }}^{x \%}, N_{\text {Selected }}^{x \%}, N_{\text {Selected }}^{x \%}$ and $N_{\text {Total }}$ are the number of true positives, the number of selected candidates at top $x \%$ screened candidates, the number of active compounds, and the total number of compounds in the screened library, respectively.

For a fair comparison with the machine-learningbased scoring function (RF-Score-VS [54]) trained on the DUD-E dataset, we selected protein targets from the DEKOIS 2.0 benchmark [55] if it has sequence identity less than $95 \%$ to any protein in the DUD-E according to the BLAST [56]. Finally, 55 targets were kept and sorted by their sequence identity, as detailed in Additional file 3: Table S3.

\section{Results and discussion The DLIGAND2 potential}

Different from the united mol2 atom type used by DLIGAND, the improved version DLIGAND2 has employed residue-specific types for protein atoms, which expanded atom types from 12 types to 169 atom types. Sufficient statistics for this larger number of atom types is ensured by using 12,450 protein chains for training. Residue-specific atom types enable the discrimination of the properties (e.g. partial charge) and surrounding environments of atoms. As shown in Fig. 1a, the potential energy between ligand atom S.3 and the main-chain O atom of ASP is significantly lower than between the atom and the mainchain O atom of ARG likely because S.3 atom has a weak but negative partial charge, which is repulsive to the negative charged ASP but attractive to the positive charged ARG residue. By comparison, DLIGAND provides an average potential over 20 amino acids. Significant differences also exist for interactions involving non-polar atoms. As shown in Fig. 1b, the CB atom of GLU and the CE atom of LYS belong to C.3 as defined in mol2, despite their very different electrostatic and steric environment. Their interactions with the ligand type N.am are very different when derived independently (DLIGAND2), and enclose the average energy function from DLIGAND. 

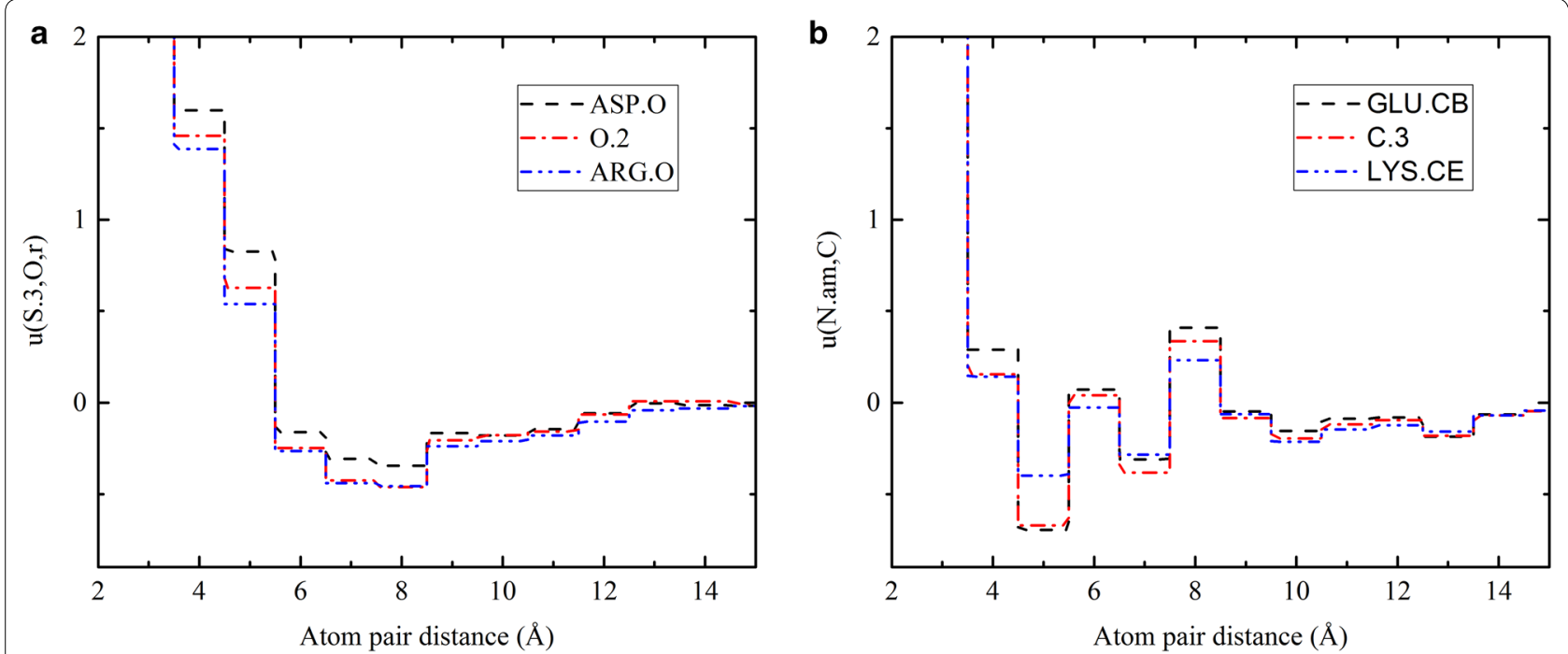

Fig. 1 The atomic interaction potentials a between ligand type S.3 and main-chain O atom of ASP, or ARG in DLIGAND2, or their common mol2 atom type (O.2) by the DLIGAND, and $\mathbf{b}$ between ligand type N.am and atom CB of GLU, or CE of LYS, or their common mol2 type "C.3" by DLIGAND, as a function of distance
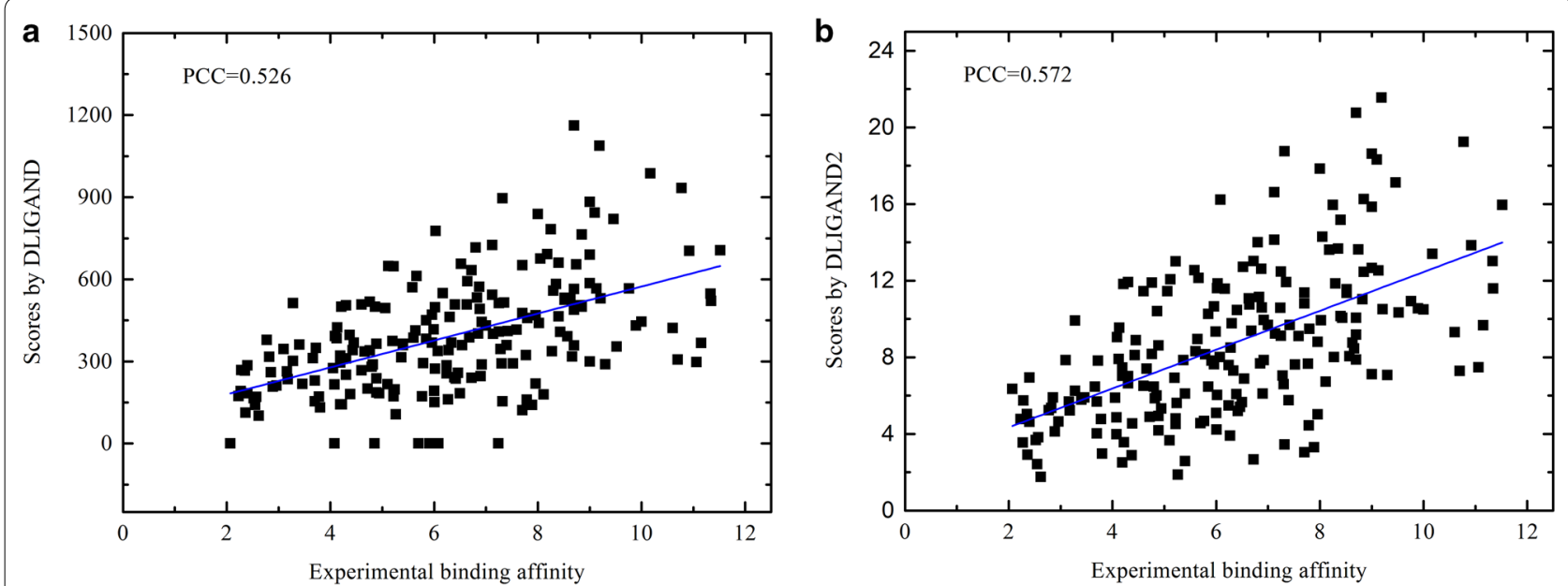

Fig. 2 Comparison between theoretically predicted and experimentally measured protein-ligand binding free energies for 195 complexes on the CASF-2013 testing set for $\mathbf{a}$ DLIGAND with a correlation coefficient of 0.526 and $\mathbf{b}$ DLIGAND2 with a correlation coefficient of 0.572 . The solid line is from the regression fit

\section{Evaluation results on CASF-2013 benchmark \\ Score power}

Figure 2 compares DLIGAND and DLIGAND2 in term of their ability for predicting protein-ligand binding affinity using the CASF-2013 dataset. DLIGAND2 achieves a higher Pearson correlation coefficient (PCC) (0.572) than DLIGAND (0.526). Table 1 further compares PCC values given by 29 other scoring functions. DLIGAND2 ranks the 9th among 30 scoring functions. The improvement of DLIGAND2 over DLIGAND was made without additional training. Interestingly, the top five scores (RF-Score-v2, ID-Score, $\Delta$ vinaRF ${ }_{20}$, AutoDock-hybrid and $\mathrm{X}$-Score ${ }^{\mathrm{HM}}$ ) were all trained directly for binding affinity prediction. The scoring function of Autodock Vina achieves a PCC of 0.56, which is lower than DLIGAND2 but higher than DLIGAND. According to the root mean square error (RMSE), DLIGAND2 (RMSE of 1.85) ranks the 10th after ChemPLP@GOLD (RMSE of 1.84), which is the best in all knowledgebased potential functions. The improvement in correlation coefficients is encouraging as DLIGAND2 was trained on protein structures only. 
Table 1 Comparisons of 30 scoring functions on the CASF-2013 dataset

\begin{tabular}{|c|c|c|c|c|}
\hline Function & PCC & RMSE & Description & Year \\
\hline RF-Score-v2 & $0.803^{a}$ & 1.54 & Machine learning & 2014 \\
\hline ID-Score & $0.753^{b}$ & 1.63 & Descriptor-based and empirical & 2013 \\
\hline$\Delta$ vinaRF $_{20}$ & $0.686^{c}$ & 1.64 & Machine learning & 2016 \\
\hline AutoDockHybrid & 0.64 & n.a. & Force fields and machine learning & 2016 \\
\hline X-Score ${ }^{\mathrm{HM}}$ & 0.614 & 1.78 & Empirical & 2002 \\
\hline$\triangle S A S A$ & 0.606 & 1.79 & Empirical & 2014 \\
\hline ChemScore@SYBYL & 0.592 & 1.82 & Empirical & 1998 \\
\hline ChemPLP@GOLD & 0.579 & 1.84 & Empirical & 2009 \\
\hline DLIGAND2 & 0.572 & 1.85 & Knowledge-based & This paper \\
\hline SMoG2016 & $0.57^{d}$ & 1.68 & Knowledge-based and empirical & 2016 \\
\hline PLP1@DS & 0.568 & 1.86 & Empirical & 2000 \\
\hline AutoDock Vina & $0.563^{e}$ & 1.87 & Knowledge-based and empirical & 2010 \\
\hline G-Score@SYBYL & 0.558 & 1.87 & Energy-based & 1997 \\
\hline ASP@GOLD & 0.556 & 1.88 & Statistical potential & 2005 \\
\hline ASE@MOE & 0.544 & 1.89 & Empirical & n.a. \\
\hline ChemScore@GOLD & 0.536 & 1.90 & Empirical & 2003 \\
\hline DLIGAND & 0.526 & 1.92 & Knowledge-based & 2005 \\
\hline D-Score@SYBYL & 0.526 & 1.92 & Energy-based & 2001 \\
\hline Alpha-HB@MOE & 0.511 & 1.94 & Empirical & n.a. \\
\hline LUDI3@DS = & 0.487 & 1.97 & Empirical & 1998 \\
\hline GoldScore@GOLD & 0.483 & 1.97 & Energy-based & 1997 \\
\hline Affinity-dG@MOE & 0.482 & 1.98 & Empirical & n.a. \\
\hline LigScore2@DS & 0.456 & 2.02 & Empirical & 2005 \\
\hline GlideScore-SP & 0.452 & 2.03 & Energy-based & 2006 \\
\hline SMoG2001 & 0.418 & 3.39 & Knowledge-based & 2001 \\
\hline Jain@DS & 0.408 & 2.05 & Empirical & 2006 \\
\hline PMF@DS & 0.364 & 2.11 & Statistical potential & 2006 \\
\hline GlideScore-XP & 0.277 & 2.18 & Energy-based & 2004 \\
\hline London-dG@MOE & 0.242 & 2.19 & Empirical & n.a. \\
\hline PMF@SYBYL & 0.221 & 2.20 & Statistical potential & 1999 \\
\hline
\end{tabular}

The results for 23 scoring functions were collected from Li [5], the results for RF-score-v2, ID-score, $\Delta$ vinaRF 20 and SMoG2016 (labeled as ${ }^{\text {a, }}$, c, d) were collected from Ballester [57], Li [20], Wang [58] and Theau [31], separately, and the results for DLIGAND2, Autodock Vina, and DLIGAND were calculated with default options by ourselves

n.a. not available

\section{Docking power}

The docking power refers to whether a scoring function can correctly identify the native ligand poses from the predicted poses. Table 2 shows the evaluation results of docking power compared to the results by $\mathrm{Li}$ et al. [5] using the same docking sets in the CASF-2013 benchmark. DLIGAND2 achieves 14\% improvement in success rate over DLIGAND in detecting native poses as the first ranked pose. Among all methods compared, DLIGAND2 has a moderate performance in term of success rates in ranking the native pose within top 1, 2, and 3 (at $45.1 \%$, $61 \%$ and $75.4 \%$, respectively). Nevertheless, DLIGAND2 ranks the second best in all knowledge-based/statistical potential scoring functions, behind ASP@GOLD, but better than PMF@SYBYL, PMF04@DS and PMF@DS. However, ASP@GOLD is not a pure statistical energy function but an empirical mix of a statistical potential with physical-based energetic terms in ChemScore@ GOLD. Thus, DLIGAND2 has the best performance for parameter-free statistical potentials.

\section{Ranking power}

The ranking power of a scoring function refers to its ability to correctly rank binders of a given target protein by their predicted binding affinities based on the poses from the crystal structures and optimized structures. Table 3 compares DLIGAND and DLIGAND2 to the evaluation results of other scoring functions of ranking 
Table 2 Success rates for the evaluation of docking power ranked by top three poses

\begin{tabular}{|c|c|c|c|}
\hline \multirow[t]{2}{*}{ Scoring function } & \multicolumn{3}{|c|}{ Success rates (\%) } \\
\hline & The top pose & Top two poses & Top three poses \\
\hline ChemPLP@GOLD & 81 & 86.7 & 89.7 \\
\hline ChemScore@GOLD & 77.9 & 83.1 & 88.2 \\
\hline GlideScore-SP & 78.5 & 85.6 & 87.7 \\
\hline ASP@GOLD & 71.8 & 81.5 & 87.2 \\
\hline LigScore2@DS & 76.9 & 84.1 & 86.7 \\
\hline PLP1@DS & 77.4 & 84.1 & 86.2 \\
\hline PLP2@DS & 74.4 & 81.5 & 86.2 \\
\hline Alpha-HB@MOE & 75.4 & 82.6 & 86.2 \\
\hline GoldScore@GOLD & 71.3 & 81 & 85.6 \\
\hline GlideScore-XP & 74.4 & 82.6 & 85.6 \\
\hline LUDI1@DS & 59 & 75.4 & 83.1 \\
\hline LUDI2@DS & 65.6 & 75.4 & 81.5 \\
\hline LigScore1@DS & 65.1 & 74.9 & 81 \\
\hline Affinity-dG@MOE & 63.1 & 74.9 & 81 \\
\hline London-dG@MOE & 59.5 & 73.8 & 78.5 \\
\hline X-Score ${ }^{H M}$ & 61 & 73.3 & 77.9 \\
\hline ChemScore@SYBYL & 59.5 & 69.2 & 75.4 \\
\hline X-Score & 59.5 & 69.2 & 75.4 \\
\hline DLIGAND2 & 45.1 & 61 & 75.4 \\
\hline $\mathrm{X}$-Score ${ }^{\mathrm{HP}}$ & 54.4 & 67.7 & 73.8 \\
\hline LUDI3@DS & 48.7 & 65.1 & 72.8 \\
\hline GScore@SYBYL & 45.1 & 61.5 & 72.3 \\
\hline X-Score $\mathrm{HS}^{\mathrm{HS}}$ & 54.4 & 66.7 & 72.3 \\
\hline Jain@DS & 48.2 & 62.1 & 70.8 \\
\hline PMF@SYBYL & 51.8 & 60 & 66.7 \\
\hline PMF04@DS & 51.8 & 62.6 & 66.2 \\
\hline ASE@MOE & 51.3 & 60 & 63.6 \\
\hline PMF@DS & 44.1 & 52.3 & 60 \\
\hline DLIGAND & 31.3 & 50.3 & 60.5 \\
\hline dSAS & 21.5 & 33.3 & 45.1 \\
\hline DScore@SYBYL & 18.5 & 29.7 & 42.6 \\
\hline
\end{tabular}

Results (excluding DLIGAND2 and DIGAND) cited from Li [5]. The RMSD value between one best-scored binding pose and the native binding pose is less than $2.0 \AA$

power collected by Li et al. [5]. A high-level success rate indicates a completely correct ranking of all members within each ligand cluster whereas a low-level success rate denotes ranking of the best as top 1 within a cluster. Again, DLIGAND2 has a small improvement over DLIGAND in high level success rates $(1.6 \%$ on crystal structures and $3 \%$ on optimized structures) but identical in low-level success rates. Compared to other statistical potentials (PMF@DF, ASP@GOLD, PMF@SYBYL), DLIGAND2 has the highest high-level success rate in crystal and optimized structures and the highest low-level success rate in optimized structures but not in crystal
Table 3 Success rates (\%) for the evaluation of ranking power ranked by high-level results on optimized structures

\begin{tabular}{|c|c|c|c|c|}
\hline \multirow[t]{2}{*}{ Score function } & \multicolumn{2}{|c|}{$\begin{array}{l}\text { Success rates (\%) } \\
\text { on crystal structures }\end{array}$} & \multicolumn{2}{|c|}{$\begin{array}{l}\text { Success rates (\%) } \\
\text { on optimized } \\
\text { structures }\end{array}$} \\
\hline & High-level & Low-level & High-level & Low-level \\
\hline $\mathrm{X}$-Score $\mathrm{HM}$ & 58.5 & 72.3 & 56.9 & 73.8 \\
\hline ChemScore@SYBYL & 53.8 & 67.7 & 52.3 & 69.2 \\
\hline D-Score@SYBYL & 49.2 & 63.1 & 52.3 & 63.1 \\
\hline LigScore1@DS & 52.3 & 61.5 & 50.8 & 63.1 \\
\hline$\triangle S A S$ & 49.2 & 67.7 & 50.8 & 69.2 \\
\hline DLIGAND2 & 50.8 & 63.1 & 49.2 & 64.6 \\
\hline PLP2@DS & 55.4 & 72.3 & 47.7 & 67.7 \\
\hline Alpha-HB@MOE & 52.3 & 66.2 & 47.7 & 64.6 \\
\hline ChemPLP@GOLD & 58.5 & 72.3 & 46.2 & 61.5 \\
\hline G-Score@SYBYL & 52.3 & 72.3 & 46.2 & 61.5 \\
\hline DLIGAND & 49.2 & 63.1 & 46.2 & 64.6 \\
\hline PMF@DS & 49.2 & 66.2 & 46.2 & 63.1 \\
\hline LUDI1@DS & 52.3 & 69.2 & 44.6 & 66.2 \\
\hline Jain@DS & 41.5 & 58.5 & 44.6 & 63.1 \\
\hline GoldScore@GOLD & 55.4 & 76.9 & 43.1 & 66.2 \\
\hline ASE@MOE & 40 & 64.6 & 43.1 & 63.1 \\
\hline London-dG@MOE & 43.1 & 60 & 40 & 60 \\
\hline ASP@GOLD & 47.7 & 72.3 & 38.5 & 60 \\
\hline Affinity-dG@MOE & 53.8 & 66.2 & 36.9 & 50.8 \\
\hline ChemScore@GOLD & 46.2 & 63.1 & 33.8 & 53.8 \\
\hline GlideScore-XP & 35.4 & 47.7 & 32.3 & 46.2 \\
\hline PMF@SYBYL & 43.1 & 61.5 & 30.8 & 53.8 \\
\hline GlideScore-SP & 43.1 & 56.9 & 21.5 & 38.5 \\
\hline
\end{tabular}

Results (excluding DLIGAND2 and DIGAND) cited from Li [5]

structures. This suggests that DLIGAND2 is less sensitive to structural changes, compared to ASP@GOLD that has the large drop in low-level success rate from crystal to optimized structures. Empirical scoring functions such as X-Score and ChemScore@SYBYL have the best performance in this test.

\section{Evaluation results on PDBbind data set}

The above benchmark study is based on experimentally determined, protein-ligand complex structures. We further tested DLIGAND2's ability to predict protein-ligand binding affinities by using predicted complex structures from docking. To remove random fluctuations, we generated 10 poses for each pair of protein and ligand by each docking method, and the highest score among 10 poses by each scoring function was used to represent the predicted binding affinity, respectively. As shown in Table 4, when scored by docking methods' own scoring functions, AutoDock Vina yields the best correlation and lowest error with experimental values (PCC of 0.501 and 
RMSE of 1.75), followed by GalaxyDock (PCC of 0.487 and RMSE of 1.75) and iDock (PCC of 0.485 and RMSE of 1.75). rDock and UCSF dock have $\mathrm{PCC}<0.2$ and RMSE $>1.95$. Low performance by rDOCK and UCSF was consistent with a previous study [4].

When re-assessed by DLIGAND2, the PCCs of predicted binding affinity consistently improve over all eight docking methods to the levels from 0.498 to 0.537 with an average of 0.523 , and the RMSE from 1.69 to 1.76 with an average of 1.71 . This indicates the main bottleneck of current docking method is the scoring function, as also disclosed in the previous study [4]. By comparison, DLIGAND can improve PCC values for five docking programs but decrease PCC values for 3 others with an average PCC of 0.455 and RMSE of 1.79 , which are $13 \%$ lower and $4.7 \%$ higher than DLIGAND2, respectively. On the basis of average value, X-Score has a performance comparable to DLIGAND2 in PCC but a slightly higher error in RMSE. It should be noted that X-Score was trained on the complex structures homologous to the CASF-2013 benchmark dataset used here, whereas DLIGNAD2 was trained only by independent monomer structures. We also noted that DLIGAND2 is about 5 times faster than X-Score, which takes 2.7 and $13.3 \mathrm{~h}$, respectively to complete this dataset (a total of 40,440 docking poses) by one CPU core of the Intel E5-2692V2 $(2.2 \mathrm{GHz})$. Here, we did not compare to RF-Score (including RF-Score-v4 [59]), $\Delta$ vinaRF $_{20}$ and ID-Score because they were trained on the PBDbind refined set.

\section{Evaluation results on DUD-E data set}

The DUD-E dataset is used to examine the ability to separate true ligands from decoys, a practically important problem in virtual screening. Here, we employed the DUD-E dataset to evaluate the screening power of scoring functions. The performance of DLIGAND and DLIGAND2 is compared to those of three top ranked scoring functions in the CASF-2013 benchmark (IDScore, $\Delta$ vinaRF 20 , and $\mathrm{X}$-Score) using the poses generated by AutoDock Vina.

As shown in the Table 5 (The detailed data can be found in Additional file 4: Table S4), DLIGAND2 achieved the best performance with an average logAUC of $10.14 \%$ and enrichment factors of 6.67 for $\mathrm{EF}_{1 \%}$. DLIGAND2 achieved an average $\mathrm{EF}_{1 \%}$ of $30 \%$ higher than Autodock Vina, 52\% and 64\% higher than DLIGAND and $\mathrm{X}$-Score, separately, and above 3 times higher than IDscore. The $\log \mathrm{AUC}$ and enrichment factors of all targets are detailed in Additional file 4: Table S4. Notably, Autodock Vina ranks the 2nd by LogAUC and the first on $\mathrm{EF}_{5 \%}$ and $\mathrm{EF}_{10 \%}$, with $\mathrm{EF}_{1 \%}$ of $26 \%$ and $86 \%$ higher than those by X-Score, and ID-Score despite the fact that they can provide higher correlation coefficients than Autodock Vina to experimental binding affinities in the CASF-2013 dataset. This is likely because ID-Score and X-Score were all trained by the PDBbind dataset that are homologous to CASF-2013 dataset. The over-training issues in empirical or machining learning based scoring functions have

Table 5 The performance of six scoring functions on the DUD-E dataset

\begin{tabular}{|c|c|c|c|c|}
\hline Scoring functions & $\log A \cup C(\%)$ & $\mathrm{EF}_{1 \%}$ & $\mathrm{EF}_{5 \%}$ & $\mathrm{EF}_{10 \%}$ \\
\hline DLIGAND2 & 10.14 & 6.67 & 3.31 & 2.55 \\
\hline AutoDock Vina & 9.96 & 5.12 & 3.41 & 2.60 \\
\hline$\triangle$ vinaRF20 & 9.00 & 6.38 & 3.41 & 2.58 \\
\hline DLIGAND & 7.61 & 4.40 & 2.74 & 2.23 \\
\hline X-Score ${ }^{H M}$ & 7.25 & 4.06 & 2.68 & 2.19 \\
\hline ID-Score & 2.47 & 1.61 & 1.42 & 1.36 \\
\hline
\end{tabular}

The highest values in each column are labeled italics

Table 4 Pearson correlation coefficients and root mean squared error between experimental binding affinity and binding affinity predicted by DLIGAND, DLIGAND2, and X-Score using docking poses generated by eight docking programs along with the results from the docking programs

\begin{tabular}{|c|c|c|c|c|c|c|c|c|}
\hline \multirow[t]{2}{*}{ Docking program } & \multicolumn{4}{|c|}{ Pearson correlation coefficient } & \multicolumn{4}{|c|}{ Root mean squared error } \\
\hline & Self & DLIGAND & DLIGAND2 & X-Score & Self & DLIGAND & DLIGAND2 & X-Score \\
\hline AutoDock & 0.404 & 0.465 & 0.537 & 0.547 & 1.91 & 1.77 & 1.69 & 1.68 \\
\hline AutoDock Vina & 0.501 & 0.459 & 0.519 & 0.536 & 1.74 & 1.78 & 1.72 & 1.69 \\
\hline rDock & 0.102 & 0.463 & 0.535 & 0.507 & 2.01 & 1.78 & 1.70 & 1.76 \\
\hline LeDock & 0.426 & 0.457 & 0.532 & 0.54 & 1.82 & 1.78 & 1.69 & 1.69 \\
\hline UCSF DOCK & 0.195 & 0.427 & 0.498 & 0.488 & 1.97 & 1.81 & 1.74 & 1.76 \\
\hline iDock & 0.485 & 0.461 & 0.522 & 0.54 & 1.75 & 1.78 & 1.71 & 1.69 \\
\hline GalaxyDock & 0.487 & 0.464 & 0.537 & 0.532 & 1.75 & 1.78 & 1.69 & 1.71 \\
\hline iGEMDOCK & 0.384 & 0.444 & 0.501 & 0.502 & 1.85 & 1.80 & 1.76 & 1.82 \\
\hline Average & 0.373 & 0.455 & 0.523 & 0.524 & 1.85 & 1.79 & 1.71 & 1.73 \\
\hline
\end{tabular}


also been observed in several previous studies [23]. The improvement of DLIGAND2 relative to Autodock Vina is more consistent in this independent test. As for RF-Score, the general version (RF-Score v3) for predicting binding affinity doesn't achieve a good performance with 5.42 for $\mathrm{EF}_{1 \%}$ [53], ranking even behind DLIGAND. Although RFScore-VS version specifically trained based on DUD-E was reported to achieve $\mathrm{EF}_{1 \%}$ values up to 38.96 [53], the per-target cross validation tends to have an over-estimate due to protein homologs between training and test sets [60]. We will employ an external DEKOIS 2.0 dataset to evaluate DLIGAND2 and RF-Score-VS separately below.

To further compare the performance of each scoring function for different protein categories, 102 targets of DUD-E dataset are separated into eight categories and evaluated by the average $\mathrm{EF}_{1 \%}$ as shown in Table 6. DLIGAND2 has the highest values of $\mathrm{EF}_{1 \%}$ in Cytochrome P450, GPCR, Kinase, and Protease. Especially in the category of GPCR and Kinase, DLIGAND2 has obvious advantages compared with other scoring functions by 2.03 times and 1.42 times better than the second ranked methods $\left(\Delta \operatorname{vinaRF}_{20}\right.$ and DLIGAND), respectively. By comparison, AutoDock Vina performs the best in the ion channel, and is far superior to DLIGAND. The scoring function $\Delta$ vinaRF 20 performs the best in miscellaneous, nuclear receptors and other enzymes. DLIGAND2 doesn't perform well in targets of kinesin-like protein 1 (KIFF11, miscellaneous) and poly (ADP-ribose) polymerase-1 (PARP1, other enzymes), likely because their binding ligand contains halogen and phosphate elements that don't appear in training protein chains. Currently, DLIGAND2 simply treats phosphate elements equivalent to the sulfate atom type. This issue may be solved in future study by including additional ligand atoms from proteinligand complex structures.

Among the best examples of DLIGAND2 performance, we plotted the receiver operating characteristic
(ROC) for the case of PTN1 protein (protein-tyrosine phosphatase 1B). As shown in Fig. 3, DLIGAND2 has the highest area under the curve (AUC) of 0.769, followed by AutoDock Vina (0.75), X-Score (0.729) and DLIGAND (0.639). The differences are more significant at lower false positive rate, the most important region for virtual screening. Indeed, the $\mathrm{EF}_{1 \%}$ are 28.89, 9.12, 18.32, 9.33 and 9.33 for DLIGAND2, Autodock Vina, $\triangle$ vinaRF20, DLIGAND and X-Score, respectively. The AUC of ID-Score is 0.553 , close to 0.5 by the random selection.

\section{Evaluation results on DEKOIS 2.0 data set}

To compare with the latest RF-Score-VS v2 (https://githu b.com/oddt/oddt) scoring function that was trained on the DUD-E, we have compiled a new dataset from the DEKOIS 2.0 benchmark, with all targets sorted according to their sequence identity to the DUD-E targets according

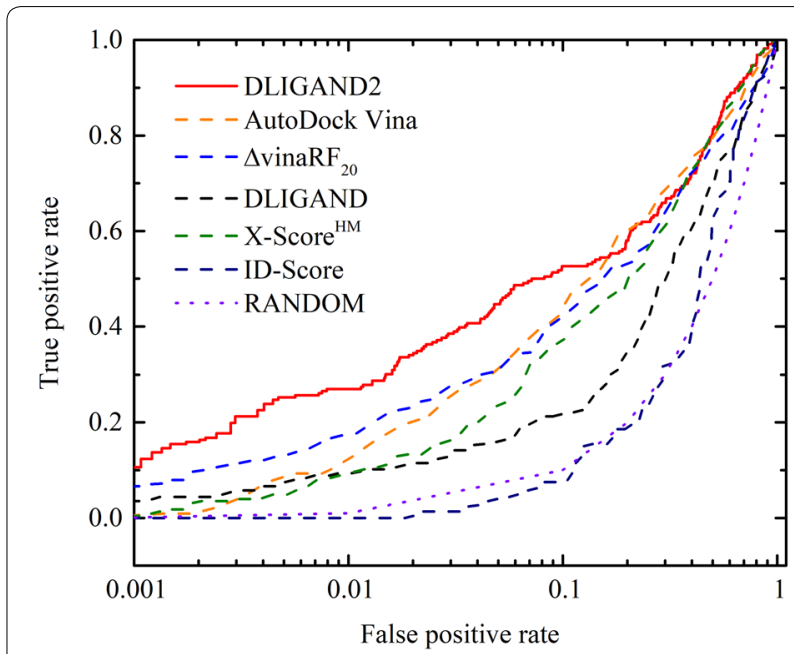

Fig. 3 Receiver operating characteristic (ROC) curves for the target PTN1 protein by different scoring methods

Table 6 Enrichment factor values $\left(\mathrm{EF}_{1 \%}\right)$ by DLIGAND2, AutoDock Vina, $\Delta$ vinaRF 20 , DLIGAND, X-Score ${ }^{\mathrm{HM}}$, ID-Score on eight protein categories

\begin{tabular}{|c|c|c|c|c|c|c|}
\hline & DLIGAND2 & Vina & $\Delta$ vinaRF $_{20}$ & DLIGAND & X-Score ${ }^{\mathrm{HM}}$ & ID-Score \\
\hline Cytochrome P450 & 6.48 & 1.93 & 3.77 & 5.10 & 3.59 & 0.56 \\
\hline GPCR & 8.46 & 2.48 & 4.17 & 3.79 & 1.56 & 1.49 \\
\hline Ion channel & 0.84 & 4.47 & 3.48 & 0.51 & 0.00 & 0.83 \\
\hline Kinase & 10.01 & 6.10 & 7.50 & 4.41 & 5.93 & 1.04 \\
\hline Miscellaneous & 6.89 & 5.65 & 7.82 & 6.63 & 4.46 & 5.36 \\
\hline Nuclear receptors & 5.42 & 9.14 & 9.57 & 4.57 & 4.01 & 1.69 \\
\hline Other enzymes & 3.23 & 3.88 & 4.76 & 2.52 & 2.64 & 1.21 \\
\hline Protease & 10.16 & 4.63 & 6.99 & 8.65 & 5.58 & 2.57 \\
\hline Average & 6.43 & 4.79 & 6.01 & 4.52 & 3.47 & 1.84 \\
\hline
\end{tabular}

Italic fonts highlight the highest value in each category 


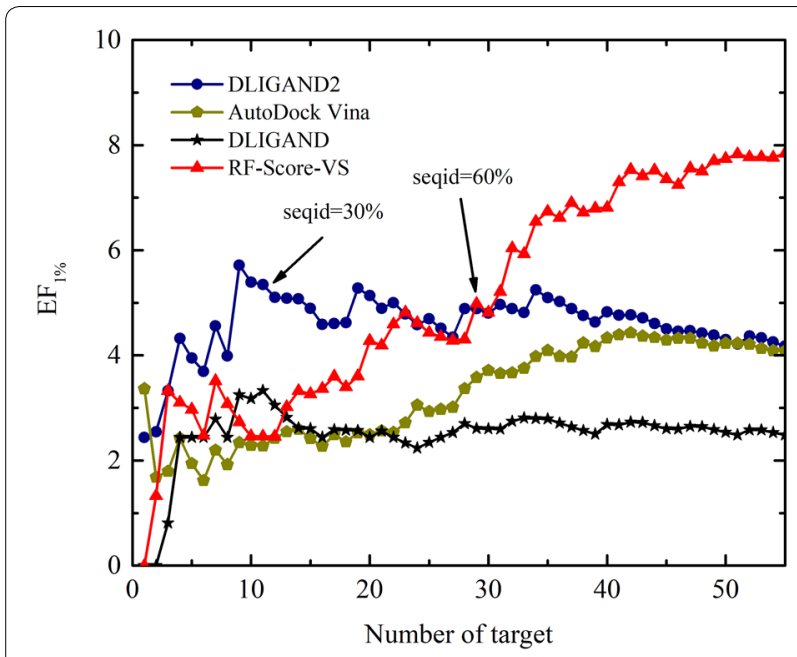

Fig. 4 The average $\mathrm{EF}_{1 \%}$ in the DEKOIS 2.0 benchmark over the number of targets sorted according to their increasing sequence identity (seqid) by blastpgp from the DUD-E targets

to the blastpgp. Figure 4 plots the average enrichment factor $\left(\mathrm{EF}_{1 \%}\right)$ as a function of the number of targets sorted according to sequence identity. The average $\mathrm{EF}_{1 \%}$ for RFScore-vs increases as the sequence identity increases, suggesting the performance of RF-Score-VS v2 is strongly depending on similarity to its training set. AutoDock Vina also has some dependence on similarity to DUD-E targets. By comparison, DLIGAND and DLIGAND2 have the least dependence except when the number of targets is low $(<10)$ likely due to natural fluctuations. DLIGAND2 has the highest performance when homologous targets are excluded for sequence identity less then $30 \%$ with an average $\mathrm{EF}_{1 \%}$ at 5.72 , compared to 2.34 by AutoDock Vina, 2.73 by RF-Score-VS and 3.25 by DLIGAND.

\section{Conclusion}

We have developed a new knowledge-based scoring function DLIGAND2 by extending to 167 atom types for protein atoms from 13 types in the original DLIGAND. Residue-specific atom types for proteins allow a more accurate description of the interaction of a ligand atom with different residues. To ensure sufficient statistics, DLIGAND2 is based on an updated non-redundant dataset of 12,450 protein chains, 62 times bigger than the dataset (195 structures) used in the original DLIGAND.

DLIGAND2 consistently improves over DLIGAND in binding affinity prediction using either native or docking-predicted complex structures. The improvement in Pearson correlation coefficient is $8.7 \%$ for the CASF-2013 dataset by using native complex structures and $15 \%$ for the PDBbind dataset by using predicted complex structures. In addition, DLIGAND2 has significantly higher enrichment than DLIGAND in discriminating true ligands from decoys using the DUD-E dataset according to re-ranking of docked structures. These results suggest the usefulness of expanding protein atomic types in generating the DLIGAND 2 statistical potential.

DLIGAND2 is the best knowledge-based energy score but not as accurate as a few empirical (X-Score) or machine-learning based (RF-Score-v2 and ID-Score) scores trained by CASF-2013 or PDBbind. The X-Score and ID-Score methods outperform Autodock vina in the CASF-2013 and PDBbind, but they all have lower performance in decoy discrimination, a practically more important problem. We have also shown that the performance of RF-score-vs strongly depends on the sequence identity of the target protein to the dataset for training the method. Though RF-score-vs was reported to perform well in the DUD-E that includes many homologous proteins to its training set, it doesn't perform well on protein targets that are not homologous to its training set. By comparison, DLIGAND2 was derived from only protein monomer structures, ensuring a balanced performance for all targets. Considering the simplicity and fast computation, DLIGAND2 will be useful for re-scoring after docking, or being included as a term for other scoring functions.

\section{Additional files}

Additional file 1: Table S1. Showed $13 \mathrm{~mol} 2$ atom types mapped to 167 residue-specific atom types for protein atoms.

Additional file 2: Table S2. It contained the list of the 4044 complexes collected from PDBind refined data set.

Additional file 3: Table S3. The $\mathrm{EF}_{1 \%}$ values of four scoring functions on DEKOIS 2.0 data set are shown.

Additional file 4: Table S4. The logAUC and EF (1\%, 5\% and $10 \%)$ values of six scoring functions on the DUD-E dataset are listed.

\section{Acknowledgements}

This project was supported in part by the National Key R\&D Program of China (2018YFC0910500, 2017YFB0202600), GD Frontier \& Key Tech, Innovation Program (2015B010109004, 2018B010109006, 2019B020228001), the National Natural Science Foundation of China (U1611261, 61772566, 81801132), Guangdong Province Key Area R\&D Program (2019B010940001) and the program for Guangdong Introducing Innovative and Entrepreneurial Teams (2016ZT06D211), as well as by Australian Research Council DP180102060 and National Health and Medical Research Council (1121629) of Australia to Y.Z.

\section{Authors' contributions}

YDY, HYZ, and YQZ coordinated and managed this project. PC implemented the scoring function and conducted the experiments with assistance from YBK, YTL, YFD, JHL, HY. All authors read and approved the final manuscript. 
Received: 2 April 2019 Accepted: 27 July 2019

Published online: 07 August 2019

\section{References}

1. Manglik A, Lin H, Aryal DK, Mccorvy JD, Dengler D, Corder G, Levit A, Kling RC, Bernat V, HuBner H (2016) Structure-based discovery of opioid analgesics with reduced side effects. Nature 537:185-190

2. Valasani KR, Vangavaragu JR, Day VW, Yan SS (2014) Structure based design, synthesis, pharmacophore modeling, virtual screening, and molecular docking studies for identification of novel cyclophilin D inhibitors. J Chem Inf Model 54:902-912

3. Singh AN, Baruah MM, Sharma N (2017) Structure based docking studies towards exploring potential anti-androgen activity of selected phytochemicals against Prostate Cancer. Sci Rep 7:1955

4. Wang Z, Sun H, Yao X, Li D, Xu L, Li Y, Tian S, Hou T (2016) Comprehensive evaluation of ten docking programs on a diverse set of protein-ligand complexes: the prediction accuracy of sampling power and scoring power. Phys Chem Chem Phys 18:12964-12975

5. LiY, Han L, Liu Z, Wang R (2014) Comparative assessment of scoring functions on an updated benchmark: 2. Evaluation methods and general results. J Chem Inf Model 54:1717-1736

6. Li Y, Liu Z, Li J, Han L, Liu J, Zhao Z, Wang R (2014) Comparative assessment of scoring functions on an updated benchmark: 1. Compilation of the test set. J Chem Inf Model 54:1700-1716

7. Liu J, Wang R (2015) On classification of current scoring functions. J Chem Inf Model 55:475-482

8. Aqvist J, Medina C, Samuelsson JE (1994) A new method for predicting binding affinity in computer-aided drug design. Protein Eng 7:385-391

9. Martin AF, Brandsdal BRO, Johan A (2010) Binding affinity prediction with different force fields: examination of the linear interaction energy method. J Comput Chem 25:1242-1254

10. Carlson HA, Jorgensen WL (1995) An extended linear response method for determining free energies of hydration. J Phys Chem 99:10667-10673

11. Hou T, Wang J, Li Y, Wang W (2011) Assessing the performance of the MM/PBSA and MM/GBSA methods. 1. The accuracy of binding free energy calculations based on molecular dynamics simulations. J Chem Inf Model 51:69-82

12. Hou T, Wang J, Li Y, Wei W (2011) Assessing the performance of the MM/ PBSA and MM/GBSA methods: II. The accuracy of ranking poses generated from docking. J Comput Chem 32:866-877

13. Sun H, Li Y, Tian S, Xu L, Hou T (2014) Assessing the performance of MM/ PBSA and MM/GBSA methods. 4. Accuracies of MM/PBSA and MM/GBSA methodologies evaluated by various simulation protocols using PDBbind data set. Phys Chem Chem Phys 16:16719-16729

14. Eldridge MD, Murray CW, Auton TR, Paolini GV, Mee RP (1997) Empirical scoring functions: I. The development of a fast empirical scoring function to estimate the binding affinity of ligands in receptor complexes. J Comput Aided Mol Des 11:425-445

15. Murray CW, Auton TR, Eldridge MD (1998) Empirical scoring functions. II. The testing of an empirical scoring function for the prediction of ligandreceptor binding affinities and the use of Bayesian regression to improve the quality of the model. J Comput Aided Mol Des 12:503-519

16. Wang R, Lai L, Wang S (2002) Further development and validation of empirical scoring functions for structure-based binding affinity prediction. J Comput Aided Mol Des 16:11-26

17. Friesner RA, Banks JL, Murphy RB, Halgren TA, Klicic JJ, Mainz DT, Repasky MP, Knoll EH, Shelley M, Perry JK, Shaw DE, Francis P, Shenkin PS (2004) Glide: a new approach for rapid, accurate docking and scoring. 1. Method and assessment of docking accuracy. J Med Chem 47:1739-1749

18. Halgren TA, Murphy RB, Friesner RA, Beard HS, Frye LL, Pollard WT, Banks $J \mathrm{~L}$ (2004) Glide: a new approach for rapid, accurate docking and scoring 2. Enrichment factors in database screening. J Med Chem 47:1750-1759

19. Ballester PJ, Mitchell JBO (2010) A machine learning approach to predicting protein-ligand binding affinity with applications to molecular docking. Bioinformatics 26:1169-1175

20. Li GB, Yang LL, Wang WJ, Li LL, Yang SY (2013) ID-Score: a new empirical scoring function based on a comprehensive set of descriptors related to protein-ligand interactions. J Chem Inf Model 53:592-600
21. Li L, Khanna M, Jo I, Wang F, Ashpole NM, Hudmon A, Meroueh SO (2011) Target-specific support vector machine scoring in structurebased virtual screening: computational validation, in vitro testing in kinases, and effects on lung cancer cell proliferation. J Chem Inf Model 51:755-759

22. Zheng S, Li Y, Chen S, Xu J, Yang Y (2019) Predicting drug protein interaction using quasi-visual question answering system. http://bioRx iv.org/abs/588178

23. Gabel J, Desaphy J, Rognan D (2014) Beware of machine learningbased scoring functions-on the danger of developing black boxes. J Chem Inf Model 54:2807-2815

24. DeWitte RS, Shakhnovich El (1996) SMoG: de novo design method based on simple, fast, and accurate free energy estimates. 1. Methodology and supporting evidence. J Am Chem Soc 118:11733-11744

25. Grzybowski BA, Ishchenko AV, Shimada J, Shakhnovich El (2002) From knowledge-based potentials to combinatorial lead design in silico. Acc Chem Res 35:261-269

26. Velec HFG, Gohlke H, Klebe G (2005) DrugScore ${ }^{\text {CSD }}$ Knowledge-Based Scoring Function derived from small molecule crystal data with superior recognition rate of near-native ligand poses and better affinity prediction. J Med Chem 48:6296-6303

27. Huang S, Zou X (2006) An iterative knowledge-based scoring function to predict protein-ligand interactions: I. Derivation of interaction potentials. J Comput Chem 27:1866-1875

28. Huang S, Zou X (2006) An iterative knowledge-based scoring function to predict protein-ligand interactions: II. Validation of the scoring function. J Comput Chem 27:1876-1882

29. Mooij WTM, Verdonk ML (2005) General and targeted statistical potentials for protein-ligand interactions. Proteins 61:272-287

30. Neudert G, Klebe G (2011) DSX: a knowledge-based scoring function for the assessment of protein-ligand complexes. J Chem Inf Model 51:2731-2745

31. Debroise T, Shakhnovich El, Chéron N (2017) A hybrid knowledgebased and empirical scoring function for protein-ligand interaction: SMoG2016. J Chem Inf Model 57:584-593

32. Huang S, Zou X (2010) Inclusion of solvation and entropy in the knowledge-based scoring function for protein-ligand interactions. J Chem Inf Model 50:262-273

33. Zhou H, Zhou Y (2002) Distance-scaled, finite ideal-gas reference state improves structure-derived potentials of mean force for structure selection and stability prediction. Protein Sci 11:2714-2726

34. Yang Y, Zhou Y (2008) Ab initio folding of terminal segments with secondary structures reveals the fine difference between two closely related all-atom statistical energy functions. Protein Sci 17:1212-1219

35. Zhao H, Yang Y, Zhou Y (2010) Structure-based prediction of DNA-binding proteins by structural alignment and a volume-fraction corrected DFIRE-based energy function. Bioinformatics 26:1857-1863

36. Zhao H, Yang Y, Zhou Y (2011) Structure-based prediction of RNAbinding domains and RNA-binding sites and application to structural genomics targets. Nucleic Acids Res 39:3017-3025

37. Zhao H, Yang Y, von Itzstein M, Zhou Y (2014) Carbohydrate-binding protein identification by coupling structural similarity searching with binding affinity prediction. J Comput Chem 35:2177-2183

38. Zhang C, Liu S, Zhu QQ, Zhou YQ (2005) A knowledge-based energy function for protein-ligand, protein-protein, and protein-DNA complexes. J Med Chem 48:2325-2335

39. Hanson J, Paliwal K, Litfin T, Yang Y, Zhou Y (2018) Accurate prediction of protein contact maps by coupling residual two-dimensional bidirectional long short-term memory with convolutional neural networks. Bioinformatics 34:4039-4045

40. Xu B, Yang Y, Liang H, Zhou Y (2010) An all-atom knowledge-based energy function for protein-DNA threading, docking decoy discrimination, and prediction of transcription-factor binding profiles. Proteins Struct Funct Bioinform 76:718-730

41. Wang R, Fang X, Yipin LuA, Wang S (2004) The PDBbind database: collection of binding affinities for protein-ligand complexes with known three-dimensional structures. J Med Chem 47:2977-2980

42. Morris GM, Huey R, Lindstrom W, Sanner MF, Belew RK, Goodsell DS, Olson AJ (2009) AutoDock4 and AutoDockTools4: automated docking with selective receptor flexibility. J Comput Chem 30:2785-2791 
43. Trott O, Olson AJ (2010) AutoDock Vina: improving the speed and accuracy of docking with a new scoring function, efficient optimization, and multithreading. J Comput Chem 31:455-461

44. Ruizcarmona S, Alvarezgarcia D, Foloppe N, Garmendiadoval AB, Juhos S, Schmidtke P, Barril X, Hubbard RE, Morley SD (2014) rDock: a fast, versatile and open source program for docking ligands to proteins and nucleic acids. PLoS Comput Biol 10(4):e1003571

45. Zhao H, Caflisch A (2013) Discovery of ZAP70 inhibitors by high-throughput docking into a conformation of its kinase domain generated by molecular dynamics. Bioorg Med Chem Lett 23:5721-5726

46. Jiang L, Rizzo RC (2015) Pharmacophore-based similarity scoring for DOCK. J Phys Chem B 119:1083-1102

47. Li H, Leung KS, Wong MH (2012) idock: a multithreaded virtual screening tool for flexible ligand docking. In: IEEE symposium on computational intelligence in bioinformatics \& computational biology. pp 77-84

48. Baek M, Shin WH, Chung HW, Seok C (2017) GalaxyDock BP2 score: a hybrid scoring function for accurate protein-ligand docking. J Comput Aided Mol Des 31:1-14

49. Shin WH, Kim JK, Kim DS, Seok C (2013) GalaxyDock2: protein-ligand docking using beta-complex and global optimization. J Comput Chem 34:2647-2656

50. Yang JM, Chen CC (2004) GEMDOCK: a generic evolutionary method for molecular docking. Proteins Struct Funct Bioinform 55:288-304

51. Mysinger MM, Carchia M, Irwin JJ, Shoichet BK (2012) Directory of useful decoys, enhanced (DUD-E): better ligands and decoys for better benchmarking. J Med Chem 55:6582

52. Litfin T, Zhou YQ, Yang YD (2017) SPOT-ligand 2: improving structurebased virtual screening by binding-homology search on an expanded structural template library. Bioinformatics 2017:1238-1240
53. Yang Y, Zhan J, Zhou Y (2016) SPOT-ligand: fast and effective structurebased virtual screening by binding homology search according to ligand and receptor similarity. J Comput Chem 37:1734-1739

54. Wójcikowski M, Ballester PJ, Siedlecki P (2017) Performance of machinelearning scoring functions in structure-based virtual screening. Sci Rep 7:46710

55. Bauer MR, Ibrahim TM, Vogel SM, Boeckler FM (2013) Evaluation and optimization of virtual screening workflows with DEKOIS 2.0 - a public library of challenging docking benchmark sets. J Chem Inf Model 53:1447-1462

56. Altschul SF, Madden TL, Schäffer AA, Zhang J, Zhang Z, Miller W, Lipman DJ (1997) Gapped BLAST and PSI-BLAST: a new generation of protein database search programs. Nucleic Acids Res 25:3389-3402

57. Ballester PJ, Schreyer A, Blundell TL (2014) Does a more precise chemical description of protein-ligand complexes lead to more accurate prediction of binding affinity? J Chem Inf Model 54:944-955

58. Wang C, Zhang Y (2016) Improving scoring-docking-screening powers of protein-ligand scoring functions using random forest. J Comput Chem 2017:169-177

59. Li H, Leung KS, Wong MH, Ballester PJ (2016) Correcting the impact of docking pose generation error on binding affinity prediction. BMC Bioinform 17:308

60. Folkman L, Stantic B, Sattar A, Zhou Y (2016) EASE-MM: sequence-based prediction of mutation-induced stability changes with feature-based multiple models. J Mol Biol 428:1394-1405

\section{Publisher's Note}

Springer Nature remains neutral with regard to jurisdictional claims in published maps and institutional affiliations.
Ready to submit your research? Choose BMC and benefit from:

- fast, convenient online submission

- thorough peer review by experienced researchers in your field

- rapid publication on acceptance

- support for research data, including large and complex data types

- gold Open Access which fosters wider collaboration and increased citations

- maximum visibility for your research: over $100 \mathrm{M}$ website views per year

At $\mathrm{BMC}$, research is always in progress.

Learn more biomedcentral.com/submissions 